\title{
Deploying an artificial intelligence-based online search tool to increase patients' access to and understanding of solid tumor gastrointestinal clinical trials
}

\author{
Emily Rose Jordan ${ }^{1 *}$, Luca Jahreiss ${ }^{1 *}$, Pashtoon Murtaza Kasi ${ }^{2,3}$ \\ ${ }^{1}$ Ancora.ai AG, Zurich, Switzerland; ${ }^{2}$ Division of Internal Medicine, Department of Hematology and Oncology, Weill Cornell Medicine, New York, \\ NY, USA; ${ }^{3}$ Englander Institute of Precision Medicine, Meyer Cancer Center, New York, NY, USA \\ Contributions: (I) Conception and design: All authors; (II) Administrative support: L Jahreiss; (III) Provision of study materials or patients: PM Kasi; \\ (IV) Collection and assembly of data: ER Jordan, L Jahreiss; (V) Data analysis and interpretation: ER Jordan, L Jahreiss; (VI) Manuscript writing: All \\ authors; (VII) Final approval of manuscript: All authors. \\ "These authors contributed equally to this work. \\ Correspondence to: Pashtoon Murtaza Kasi, MD, MS. Division of Internal Medicine, Department of Hematology and Oncology, Weill Cornell \\ Medicine, 1305 York Ave, 12th Floor, New York, NY 10044, USA. Email: pmk4001@med.cornell.edu.
}

Background: The number and complexity of clinical trials is growing, making finding and accessing trials increasingly challenging. A key barrier to increasing recruitment efficiency is patients' low awareness of trials as an option. They rarely hear about trials from oncologists, unless these are based at a research center, which introduces trial population biases. Even if patients decide to search for trial information on their own, it is difficult for laypeople to understand. Trial search tools exist, but have multiple shortcomings: complex navigation, limited search functionality, data too complex for non-specialists, and next steps unclear.

Methods: Access to trials via a novel trial search tool using technology, including artificial intelligence (AI), to restructure trial information and match patients to trials was the focus of this study. We focused on 6 gastrointestinal cancers for evaluating the search tool's AI model in which their trial information was restructured based on key eligibility differentiators identified. Research participants were recruited with the support of patient associations leveraging social media. They were asked to complete a survey evaluating an established tool and the novel tool to find trials relevant for them. They assessed aspects of the tools' usability on 5-level Likert scales.

Results: Survey respondents had a base level of awareness of trials, with patient associations and the internet as leading information sources. The novel tool made it easier to find trials, led to an improvement in ease of understanding information presented and provided more clarity on what the next steps towards enrollment would be. Overall, this led to higher patient satisfaction.

Conclusions: Novel patient-focused tools can unlock trial access for all patients. Democratizing trial information will not only increase trial accrual, but also patient satisfaction while reducing disparities. This tool could also be of value for academic or community oncologists who face similar issues when navigating trials for patients.

Keywords: Artificial intelligence (AI); patient access; health equity; cancer disparities; clinical trials

Submitted Apr 14, 2021. Accepted for publication Aug 05, 2021.

doi: 10.21037/jgo-21-175

View this article at: https://dx.doi.org/10.21037/jgo-21-175 
Table 1 Current data structure in trial search tools

\begin{tabular}{lccccc}
\hline Trial search tool & By sponsor & By trial phase & By tumor & By cancer stage & Across solid tumors \\
\hline Clinicaltrials.gov & $\mathrm{x}$ & $\mathrm{x}$ & $\mathrm{x}$ & & $\mathrm{x}$ \\
Cancertrialsearch.com & $\mathrm{x}$ & $\mathrm{x}$ & $\mathrm{x}$ & $\mathrm{x}$ & $\mathrm{x}$ \\
Research centers & & & $\mathrm{x}$ & $\mathrm{x}$ \\
Patient support sites & & $\mathrm{x}$ & $\mathrm{x}$ & & Partially \\
\hline
\end{tabular}

\section{Introduction}

The number and complexity of clinical studies in oncology has continuously been growing (1). With cancer treatments becoming more personalized (including the increased availability of genetic information) and more tumoragnostic, this trend is only accelerating. Hence, getting an overview of active trials, finding relevant trials and accessing them is becoming more challenging. Barriers for clinical trial recruitment lead to considerable delays for getting innovative treatments to patients. Low awareness of trials as an option amongst patients has been identified as a key driver for this low recruitment efficiency $(2,3)$. Patients rarely hear about trials from their oncologists [indeed only $22 \%$ of patients do (4)], unless they are based at a research center. This introduces trial population biases, as only a small subset of patients has access to these specialists. Even if patients decide to search for information on trials themselves, the tools and information currently available are difficult to understand, use and cross-compare. There are existing tools that support patients searching for trial options, but they have multiple shortcomings: complex navigation, limited search functionality $(3,5)$, clinical trial data too complex for non-specialists to understand (6), and lack of clarity on next steps (5).

We present the following article in accordance with the GRIPP2 reporting checklist (available at https://dx.doi. org/10.21037/jgo-21-175).

\section{Methods}

The main focus of this study was assessing a novel trial search tool (www.cancertrialsearch.com) to provide information on and access to clinical trials for patients. This tool uses natural language processing, a specific form of artificial intelligence, to restructure trial information to enable efficient search through trial options and automated identification of relevant trials for an individual patient. For the purpose of this study, six of the most commonly occurring gastrointestinal (GI) cancers in the United States (US) (cholangiocarcinoma, colorectal cancer, esophageal cancer, gallbladder cancer, liver cancer, pancreatic cancer, stomach cancer) were chosen for inclusion in this study. This was arbitrarily done keeping in line with common GI cancers and to maintain a homogeneous patient population. Listings and data for all trials in these indications in the ClinicalTrials.org registry were extracted, restructured and standardized using the natural language processing models mentioned above, with a focus on trial eligibility criteria. The data was refreshed weekly during the course of the study to ensure full trial coverage for participants. Patients interfaced with the novel search tool through a free, online website (www.cancertrialsearch.com) written in patient-friendly language and available in English and Spanish. Patients then answered a short, targeted questionnaire customized for each cancer. The cancerspecific questionnaire focused on key eligibility criteria that were identified as differentiators between single trials to help patients quickly match to relevant trials (Table 1).

Once the tool was designed, social media and market research surveys were utilized to get feedback on the platform. Twenty-nine participants (GI oncology patients) across the US were recruited with the support of patient associations and advocates (see Acknowledgments). As a comparator, we also recruited an independent cohort of 35 non-GI oncology patients using the same techniques.

Participants were asked to complete a 20 -minute online survey built in Google Forms evaluating both an established tool (clinicaltrials.gov) and the novel tool to find trials relevant for them. Consent was provided after reading a short description of the study objectives, methods and consent policy. To reduce bias, they were only provided with basic instructions on how to use the tools and were instructed to look for trials relevant for themselves and select one of them to review in more detail by clicking through to the full trial listing from the results page. The sequence of the appearance of the tools in the questionnaire 
Table 2 Distribution of respondents across cancer types

\begin{tabular}{lcc}
\hline Cancer type & Respondents & $\begin{array}{c}\text { US incident } \\
\text { population (7) }\end{array}$ \\
\hline Colorectal cancer & $64 \%$ & $48 \%$ \\
Esophageal cancer & $7 \%$ & $6 \%$ \\
$\begin{array}{l}\text { Gallbladder cancer \& } \\
\text { cholangiocarcinoma }\end{array}$ & $7 \%$ & $4 \%$ \\
Liver cancer & $0 \%$ & $14 \%$ \\
Pancreatic cancer & $11 \%$ & $19 \%$ \\
Stomach cancer & $11 \%$ & $9 \%$ \\
\hline
\end{tabular}

Table 3 Distribution of respondents by ethnicity

\begin{tabular}{lcc}
\hline Self-reported ethnicity & Respondents & US census (8) \\
\hline $\begin{array}{l}\text { American Indian or Alaska } \\
\text { Native }\end{array}$ & $6 \%$ & $1 \%$ \\
Asian & $18 \%$ & $6 \%$ \\
Black or African American & $6 \%$ & $13 \%$ \\
Caucasian & $65 \%$ & $60 \%$ \\
$\begin{array}{l}\text { Hispanic or Latino or Spanish } \\
\text { Origin }\end{array}$ & $6 \%$ & $19 \%$ \\
Native Hawaiian or Other & $0 \%$ & $0 \%$ \\
Pacific Islander & & $0 \%$ \\
Prefer not to say & $6 \%$ & \\
\hline
\end{tabular}

Table 4 Distribution of respondents by education level

\begin{tabular}{lcc}
\hline Education level & Respondents & US census (9) \\
\hline High school & $16 \%$ & $30 \%$ \\
Some college & $11 \%$ & $23 \%$ \\
Associate's degree & $11 \%$ & $10 \%$ \\
Bachelor's degree & $26 \%$ & $23 \%$ \\
$\begin{array}{l}\text { Graduate or professional } \\
\text { degree }\end{array}$ & $37 \%$ & $14 \%$ \\
\hline
\end{tabular}

was randomized to ensure unbiased answers. After using each tool, they had to assess different aspects of the tools' usability on a 5 -level Likert scale.

\section{Statistical analysis}

Survey results were analyzed (Microsoft Excel,
Table 5 Distribution of respondents by age group

\begin{tabular}{lcc}
\hline Age group & Respondents & US CRC incidence (10) \\
\hline$<18$ & $0 \%$ & $0 \%$ \\
$18-24$ & $7 \%$ & $1 \%$ \\
$25-34$ & $29 \%$ & $1 \%$ \\
$35-44$ & $39 \%$ & $5 \%$ \\
$45-54$ & $14 \%$ & $15 \%$ \\
$55-64$ & $11 \%$ & $22 \%$ \\
$65+$ & $0 \%$ & $56 \%$ \\
\hline
\end{tabular}

CRC, colorectal cancer.

RRID:SCR_016137), using built-in statistical functions (mean, standard deviation, Student's $t$-test). Statistical significance with regards to the hypothesis of improved trial access leveraging the novel tool was assessed by using a paired two-tailed Student's $t$-test. Significance was defined in line with standard medical research practice (ns, $\mathrm{P}>0.05$; *, $\mathrm{P} \leq 0.05$; **, $\mathrm{P} \leq 0.01$; ***, $\mathrm{P} \leq 0.001)$.

\section{Ethical statement}

Market research surveys utilizing feedback from anonymous users were utilized with no identifiable information, hence no ethical approval by an institutional review board was required. Consent was obtained from all participants. The research was conducted in accordance with the Declaration of Helsinki (as revised in 2013).

\section{Results}

As noted, for this pilot study, 29 participants across the US were recruited with the support of patient associations and advocates participated to provide feedback on the AI tool (www.cancertrialsearch.com). They were fairly evenly distributed across different cancer types similarly to their incidence (see Table 2) (7). The respondent sample was diverse, with overrepresentation of some groups (White, Asian), but underrepresentation of others (Black, Latino), according to the most recent US census data (Table 3) (8). However, they were generally more educated than the general population ( $63 \%$ vs. $37 \%$ with bachelor's degree or higher, Table 4) (9) and younger than average GI cancer patients (majority in the 39-45 age group vs. 65+ age group, Table 5) (10). This is not surprising given selection bias in individuals using social media and online advocacy groups. 
The respondents' initial knowledge of trials varied widely (Figure 1) and they used a number of different sources to gain their existing knowledge, with patient associations and the internet the most commonly mentioned sources (Figure 2).

When comparing the novel tool against www. clinicaltrials.gov, respondents reported that trials were easier to find $(3.8 \pm 1.1$ vs. $3.2 \pm 1.2$, Figure 3$)$ and understand $(3.8 \pm 1.0$ vs. $2.9 \pm 1.4$, Figure 3$)$ using the novel tool. Moreover, once they had found a trial, the next steps to take towards enrollment on that trial were significantly clearer (3.9 \pm 1.1 vs. $3.3 \pm 1.4$, Figure 3). Overall, this also saved them time $(9.6 \pm 10.0$ vs. $21.1 \pm 15.0$ minutes, Figure 4$)$ and led to

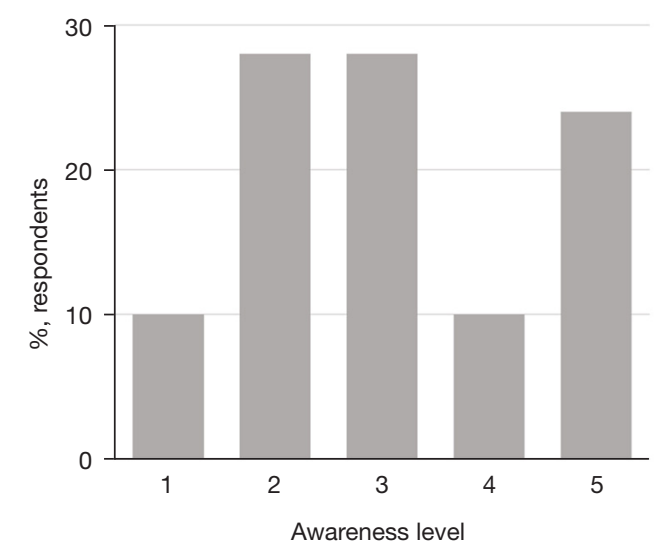

Figure 1 Wide variability of initial trial knowledge amongst participants. Participants were asked to rate "your current level of awareness of your clinical trial options on scale from 1 (not at all aware) to 5 (extremely aware)". considerably higher overall satisfaction with the trial search process $(3.7 \pm 1.2$ vs. $2.9 \pm 1.2$, Figure 3$)$. We further validated these findings in an independent cohort of non-GI cancers (94\% breast cancer, 3\% cervical cancer, 3\% prostate cancer), which showed comparable results (Figure 5).

Our study has several limitations. Firstly, it is a small pilot study with a small number of participants. Secondly, it only focuses on 6 GI cancers. Next, there is a selection bias in the participants given participation was through market research/surveys using online platforms which inherently appeals to a subset of all patients/individuals. Nevertheless, the goal of this study was to be a pilot to provide a novel problem-solving trial navigation platform using AI based tools. We showed that deploying an AI-based tool to help navigate clinical trials access is feasible and more user friendly leading to better patient satisfaction. It is available live (www.cancertrialsearch.com) and under the PlanDo-Study-Act (PDSA) cycle is something that is/can be modified and personalized to meet relevant individual or institutional needs of interest.

\section{Discussion}

Overall, the study showed that the novel patient-focused, AI-driven clinical trial support tool can streamline and help navigate trial access for all patients. More work is still needed to ensure engagement of certain subsets of the population (especially people with lower education levels and the elderly) by different outreach approaches in combination with novel tools like www.cancerclinicaltrials. com. To reach the elderly with trial information, focusing

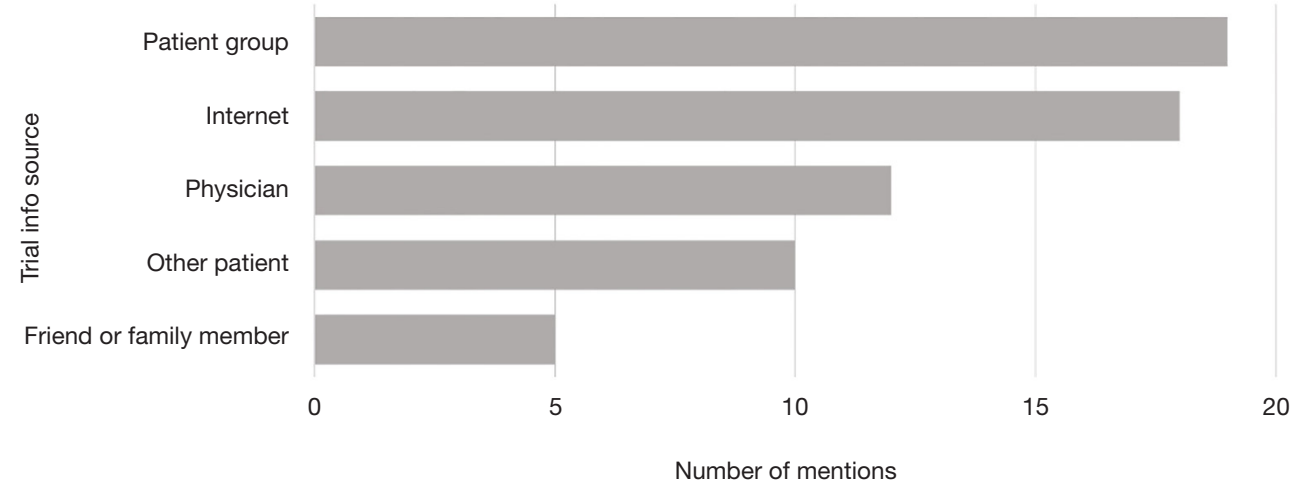

Figure 2 Patient groups and internet as most important current sources for trial information. Participants were asked to select all applicable from a given set of options, including an "other option". 


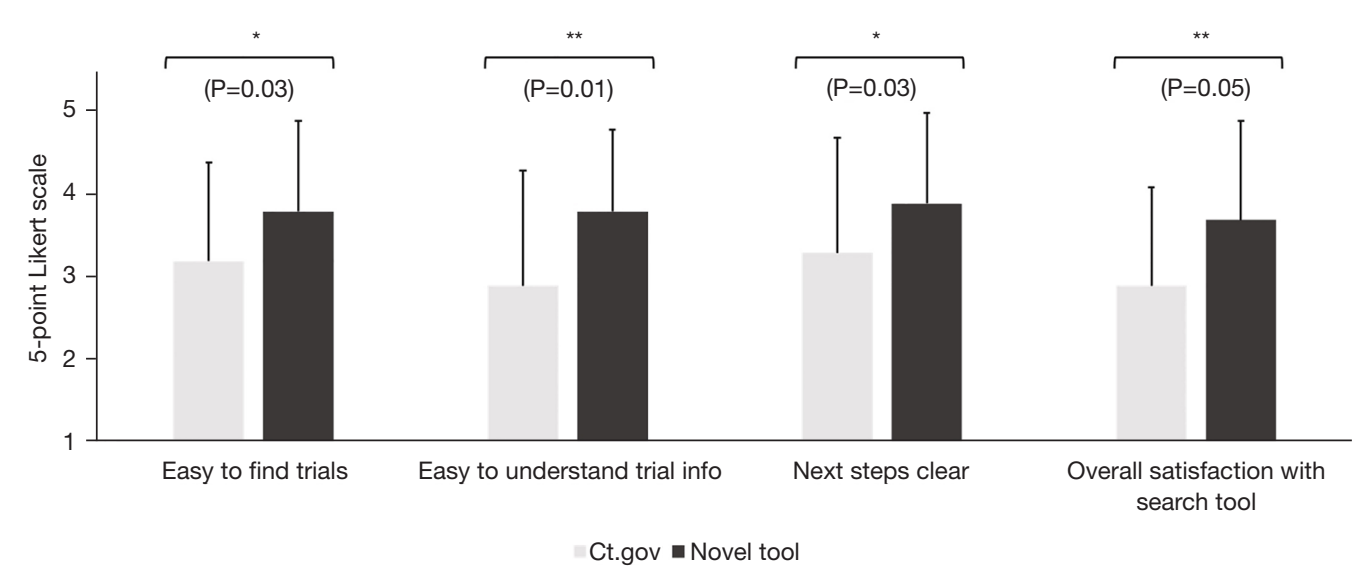

Figure 3 Increased level of performance on a number of aspects of novel tool $v$ s. currently available tool. Gastrointestinal cancer patients reviewed each tool in turn (sequence randomized) and then assessed these 4 aspects on 5 -point Likert scale. Statistical significance was assessed by using a paired two-tailed Student's $t$-test $\left.{ }^{*}, \mathrm{P} \leq 0.05 ;{ }^{* *}, \mathrm{P} \leq 0.01\right)$.

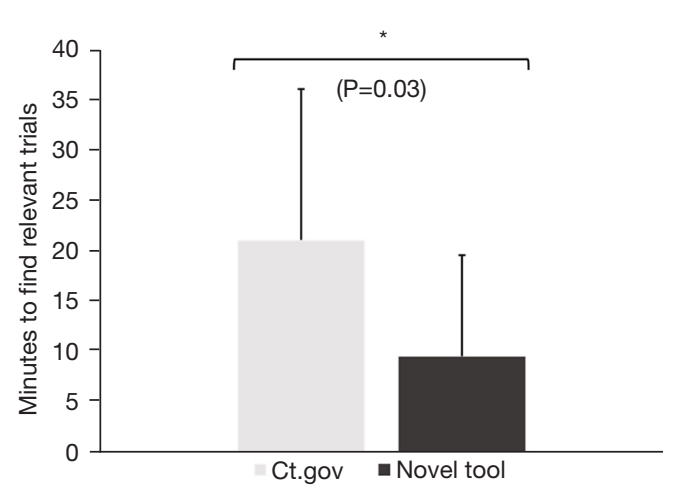

Figure 4 Faster identification of relevant trials with novel tool $v$ s. currently available tool. Participants reviewed each tool in turn and then estimated the time in minutes it took to find relevant trials. Statistical significance was assessed by using a paired two-tailed Student's $t$-test $\left(^{*}, \mathrm{P} \leq 0.05\right)$.

on younger caregivers more specifically could be a potential avenue, but will require additional research. This not only has implications for patients but also for oncologists as well. Providing community oncologists with similar tools could also help reduce the effort in finding trials and further broaden access to trials to a wider and more diverse population. Our team will be exploring AI-driven methods to improve the efficiency of physician trial search as a next step. Future directions also include involving a larger number of individuals from diverse backgrounds and disease types to provide further feedback to continually improve the tool. With the rise in liquid biopsy based genetic testing and the move towards more tumor-agnostic clinical trials, finding the most relevant trials will only become more challenging, increasing the need for an efficient way to do so for both patients and physicians. We also believe that the companies involved in developing and testing new treatments, namely pharmaceutical companies and clinical research organizations, would have an inherent interest in a broader application of such a tool, as it would increase the efficiency of the clinical trials they run and therefore allow them to bring new treatments to market faster.

\section{Acknowledgments}

We would like to thank the patient advocates and partners who helped with recruiting participants, therefore making this study possible: Colontown, Elephants and Tea, Stage 4 Pancreatic Cancer Support Group, Ticking off Breast Cancer, The Urban Kitchen, National Pancreatic Cancer Foundation, Dr. Corinne Jordan (Allina Health, Minnesota), Megan-Claire Chase, Cholangiocarcinoma Support Group, Hirshberg Foundation for Pancreatic Cancer Research, Love for Life Foundation, Living with Stomach Cancer, Young Stomach Cancer Warriors, Esophageal Cancer Patient and Caregiver Support Group. We would like to thank the ASCO Gastrointestinal Cancers Symposium for the opportunity to present initial results of this study as a poster (\#456) at the 2021 event. Citation: Kasi PM, Jordan E, Jahreiss L. Deploying an AI-based online search tool to increase patients' access to and understanding of solid tumor 


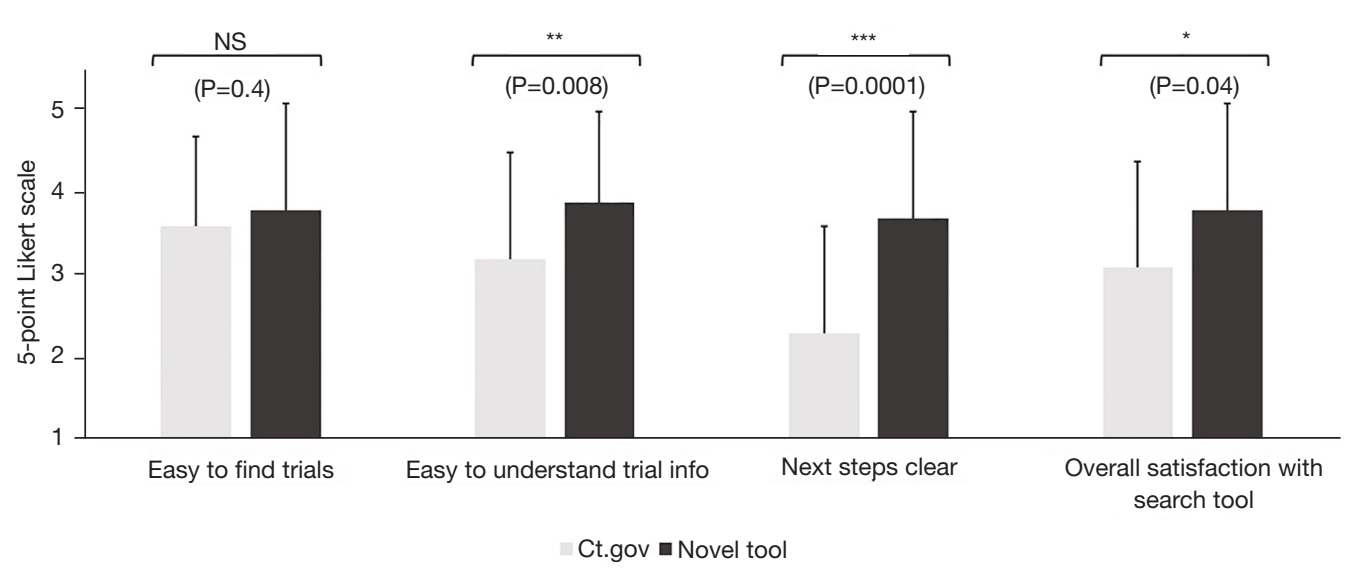

Figure 5 Increased level of performance on a number of aspects of novel tool $v$ s. currently available tool. Non-gastrointestinal cancer patients reviewed each tool in turn (sequence randomized) and then assessed these 4 aspects on 5 -point Likert scale. Statistical significance was assessed by using a paired two-tailed Student's $t$-test (ns, $\mathrm{P}>0.05 ;{ }^{*}, \mathrm{P} \leq 0.05 ;{ }^{* *}, \mathrm{P} \leq 0.01$; *** $\mathrm{P} \leq 0.001$ ).

GI clinical trials. 7 Clin Oncol 2021;39(3_suppl):abstr 456. Funding: None.

\section{Footnote}

Reporting Checklist: The authors have completed the GRIPP2 reporting checklist. Available at https://dx.doi. org/10.21037/jgo-21-175

Data Sharing Statement: Available at https://dx.doi. org/10.21037/jgo-21-175

Conflicts of Interest: All authors have completed the ICMJE uniform disclosure form (available at https://dx.doi. org/10.21037/jgo-21-175). ERJ \& LJ are current employees and shareholders of Ancora.ai AG, and have received grants from the European Union (EIT Health Headstart) and Almirall (Digital Garden Accelerator). PMK reports support not directly linked to this manuscript: (I) consultancy/ advisory board: Taiho (to institution), Ipsen (to institution), Foundation Medicine, Bayer, Axiom, Natera, Roche, IPBA, Merck, QED, Tempus Labs, Daiiche Sankyo, Boston Health Care, Delcath, Eli Lily; (II) grant funding/clinical trial support from BMS (institution), Advanced Accelerator Applications (institution), Array Biopharma (institution), Tersera (institution), Boston Scientific (institution), Celgene (institution); (III) travel accommodations: AstraZeneca (IIT presentation at Immuno-oncology conference). The authors have no other conflicts of interest to declare.
Ethical Statement: The authors are accountable for all aspects of the work in ensuring that questions related to the accuracy or integrity of any part of the work are appropriately investigated and resolved. Market research surveys utilizing feedback from anonymous users were utilized with no identifiable information, hence no ethical approval by an institutional review board was required. Consent was obtained from all participants. The research was conducted in accordance with the Declaration of Helsinki (as revised in 2013).

Open Access Statement: This is an Open Access article distributed in accordance with the Creative Commons Attribution-NonCommercial-NoDerivs 4.0 International License (CC BY-NC-ND 4.0), which permits the noncommercial replication and distribution of the article with the strict proviso that no changes or edits are made and the original work is properly cited (including links to both the formal publication through the relevant DOI and the license). See: https://creativecommons.org/licenses/by-nc-nd/4.0/.

\section{References}

1. Clinicialtrials.gov [Internet]: Trends, Charts, and Maps [cited 2021 Jan 14]. Available online: https://clinicaltrials. gov/ct2/resources/trends

2. The Need for Awareness of Clinical Research [cited 2021 Jan 14]. In: National Institutes of Health [Internet]. Bethesda. Available online: https://www.nih.gov/health- 
information/nih-clinical-research-trials-you/need-

awareness-clinical-research

3. Difficulties finding a trial to join [cited 2021 Jan 14]. In: Healthtalk.org blog [Internet]. Available online: https:// healthtalk.org/clinical-trials/difficulties-finding-a-trialto-join

4. Poll: Majority of Americans would participate in clinical trials if recommended by doctor [cited 2021 Mar 30]. In: Elsevier Connect [Internet]. Available online: https://www. elsevier.com/connect/poll-majority-of-americans-wouldparticipate-in-clinical-trials-if-recommended-by-doctor

5. Institute of Medicine (US) Forum on Drug Discovery, Development, and Translation. Transforming Clinical Research in the United States: Challenges and Opportunities: Workshop Summary. Washington (DC): National Academies Press (US); 2010. 3, Challenges in Clinical Research. Available online: https://www.ncbi.nlm. nih.gov/books/NBK50892/

Cite this article as: Jordan ER, Jahreiss L, Kasi PM. Deploying an artificial intelligence-based online search tool to increase patients' access to and understanding of solid tumor gastrointestinal clinical trials. J Gastrointest Oncol 2021;12(5):2045-2051. doi: 10.21037/jgo-21-175
6. Kang T, Elhadad N, Weng C. Initial Readability Assessment of Clinical Trial Eligibility Criteria. AMIA Annu Symp Proc 2015;2015:687-96.

7. Siegel RL, Miller KD, Fuchs H, et al. Cancer Statistics, 2021. CA Cancer J Clin 2021:71:7-33.

8. Overview of Race and Hispanic Origin: 2010 [cited 2021 Jan 14]. In: United States Census Bureau [Internet]. Available online: https://www.census.gov/prod/cen2010/ briefs/c2010br-02.pdf

9. Educational Attainment [cited 2021 Jan 14]. In: United States Census Bureau [Internet]. Available online: https:// data.census.gov/cedsci/table?q=education\&tid=ACSST 1Y2019.S1501 (Age 25 and up)

10. National Cancer Institute [Internet]: Surveillance, Epidemiology and End Results Program - Cancer Stat Facts: Colorectal Cancer [cited 2021 Jan 14]. Available online: https://seer.cancer.gov/statfacts/html/colorect.html 